\title{
Editorial
}

\section{Location-Based Mobile Marketing Innovations}

\author{
Jaegeol Yim, ${ }^{1}$ Subramaniam Ganesan, ${ }^{2}$ and Byeong Ho Kang ${ }^{3}$ \\ ${ }^{1}$ Department of Computer Engineering, Dongguk University, Gyeongju, Republic of Korea \\ ${ }^{2}$ Electrical and Computer Engineering, Oakland University, Rochester, MI, USA \\ ${ }^{3}$ School of Engineering and ICT, University of Tasmania, Hobart, TAS, Australia \\ Correspondence should be addressed to Jaegeol Yim; yim@dongguk.ac.kr
}

Received 10 July 2017; Accepted 10 July 2017; Published 12 October 2017

Copyright (C) 2017 Jaegeol Yim et al. This is an open access article distributed under the Creative Commons Attribution License, which permits unrestricted use, distribution, and reproduction in any medium, provided the original work is properly cited.

The increasing complexity of the industry means that marketers must now be experts not only in marketing but also in people, data, delivery platforms, and mobile locationbased marketing. The objective of location-based marketing via mobile devices is to encourage those activities as well as drive foot traffic, share discounts, and build customer loyalty. Mobile devices have been used to gather information about nearby businesses including reviews, directions, calling the business, and using the businesses' mobile app. With location-based mobile marketing, the business is easy to find and have skillfully combined location-based marketing with an overall targeted marketing approach that includes social media, push notifications, email newsletters, and also offline marketing.

The objective of this special issue is to bring together research contributions of unpublished research on the recent development and innovations about location-based mobile marketing (LBMM). The fundamental infrastructure of LBMM is obviously location-based service (LBS) systems. The most distinguished parts of LBS systems include positioning components and map provisioning components that are running on the general computing systems. On top of the LBS system, LBMM requires providing more intelligent marketing oriented services to customers. More and more machine learning methods, especially convolutional neural networks, have been used to analyze user behavior and classify usage patterns in development of intelligent services. With increasing model training parameters and data scales, the traditional single machine training method cannot meet the requirements of time complexity in practical application scenarios. Therefore, the data parallel model or parallel method has been employed in order to speed up the training process. The current training framework often uses simple data parallel or model parallel methods that do not fully utilize heterogeneous computing resources. The paper entitled "A Parallel Strategy for Convolutional Neural Network Based on Heterogeneous Cluster for Mobile Information System" by J. Zhang et al. proposes a delayed synchronization convolutional neural network using the heterogeneous system. This is a parallel strategy based on both synchronous parallel and asynchronous parallel approaches, and the model training process can reduce the dependence on the heterogeneous architecture in the premise of ensuring the model convergence. Therefore, the convolution neural network framework is better suited for different heterogeneous system environments.

Intelligent service is to guess desired services that the customer might want and recommend them to the customer. The SlopeOne model has been widely used in guessing desired services. The paper entitled "Collaborative QoS Prediction for Mobile Service with Data Filtering and SlopeOne Model" by Y. Yin et al. proposes a data filtering-extended SlopeOne model (based on collaborative filtering). The idea behind this model is based on the characteristics of a mobile service and the relation with location. With the model, the quality of service (QoS) values can be predicted.

As an important source of mobile location-based data, taxi mobility information can be referred to when making marketing decisions. Studying the behavioral patterns of taxis in a city during the holidays using the global positioning system (GPS) can yield remarkable insights into people's holiday travel patterns, as well as the odd-even day vehicle prohibition system. Using GPS data, the paper entitled "Exploring Intracity Taxi Mobility During Holidays for Location-Based 
Marketing" by W. Wang et al. studied the behavioral patterns of taxis during specific holidays in terms of pick-up and drop-off locations, travel distance, mobile step length, travel direction, and radius of gyration.

LBMM gathers and uses information about users' locations. Therefore, ensuring information security and privacy is one of the most important concerns of users. The paper entitled "A Hybrid Location Privacy Solution for Mobile LBS" by R. Gupta and U. P. Rao proposes a hybrid solution, HYB, to achieve location privacy for the mobile users who use location services frequently. The proposed HYB scheme is based on the collaborative preprocessing of location data and utilizes the benefits of homomorphic encryption technique. Location privacy is achieved at two levels, namely, at proximity level and at distant level. The proposed HYB solution preserves user's location privacy effectively under specific, pull-based, sporadic query scenario.

LBMM systems and as an example the sensor nodes often face various attacks where the attackers try to manipulate the estimated location or try to provide false beacons. The paper entitled "A Secure Localization Approach Using Mutual Authentication and Insider Node Validation in Wireless Sensor Networks" by G. Kumar et al. has proposed a methodology that will address this problem of security aspects in localization of the sensor nodes. Moreover, they have considered the network environment with random node deployment and mobility as these two conditions are less addressed in previous research works. Further, they proposed an algorithm that requires low overhead due to the usage of less control messages in a limited transmission range. In addition, they have also proposed an algorithm to detect the malicious anchor nodes inside the network. The paper entitled "Location Privacy Protection Based on Improved $K$ Value Method in Augmented Reality on Mobile Devices" by C. Yin et al. proposes a privacy protection method combining the $k$-anonymity with pseudonym methods and shows that the method can effectively anonymize all service requests. The paper titled "An Enhancement of Optimized Detection Rule of Security Monitoring and Control for Detection of Cyber Threat in Location-Based Mobile" by W. Park and B. H. Kang analyzes SNORT detection rules and proposes a guideline of SNORT rule optimizations to improve the efficiency and accuracy of intrusion detection operations. This enables the intrusion detection system to be more secure in location-based mobile services.

In the mobile service environment, it is required that mobile terminals should efficiently use wireless network resources. In addition, because video streaming becomes a major service among the data services of mobile terminals in heterogeneous networks, the necessity of the efficient network access control for heterogeneous wireless networks is raised as an important topic. The paper titled "Network Access Control for Location-Based Mobile Services in Heterogeneous Wireless Networks" by D.-Y. Kim et al. proposes a novel network access control in heterogeneous wireless networks. The proposed method estimates the network status with Naïve Bayesian Classifier and performs network access control according to the estimated network status. Thus, it improves data transmission efficiency to satisfy the quality of services.

Mobility management is an essential challenge for supporting reliable multimedia data streaming over wireless and mobile networks in the Internet of Things (IoT) for LBMM applications. The paper titled "Design of Optimized Multimedia Data Streaming Management Using OMDSM over Mobile Networks" by B. Park et al. introduces a new enhanced data streaming route optimization scheme that uses an optimized Transmission Control Protocol (TCP) realignment algorithm in order to prevent the packet disordering problem whenever the nodes in the IoT environment are communicating with each other. With the proposed scheme, data packets sequence realignment can be prevented, the packet traffic speed can be controlled, and the TCP performance can be improved.

In large-scale location-based services, an ideal situation is that self-adapting routing strategies use future traffic data as input to generate a topology which could adapt well to the changing traffic. The paper entitled "A Traffic Prediction Model for Self-Adapting Routing Overlay Network in Publish/Subscribe System" by M. Chi et al. proposes a traffic prediction model for the broker in publish/subscribe system, which can predict the traffic of the link in future by using neural networks.

As LBMM systems evolve and become more and more intelligent, they require more and more complicated operations and computing resources. As a result, executing them on the mobile computing systems takes too much time or becomes impossible. To overcome this problem, a computation offloading technique can be used to execute certain tasks of LBMM in cloud and fog environments. The paper entitled "Offloading Method for Efficient Use of Local Computational Resources in Mobile Location-Based Services Using Clouds" by Y. Son and Y. Lee introduces a computation offloading technique that utilizes fog computing to improve the performance of virtual machines running on mobile devices. LBMM systems make use of virtual machine technology in order to run on a variety of platforms.

LBMM systems, with most components of them running on mobile terminals with limited computing resources, should be optimized in terms of resource consumptionespecially battery power. The paper entitled "Automatic Optimizer Generation Method Based on Location and Context Information to Improve Mobile Services" by Y. Son et al. introduces a technique to automatically generate a customized service optimizer for each application, service type, and platform using location and situation information. By using the proposed technique, energy and computing resources can be more efficiently employed for each service. Thus, users should receive more effective LBSs on mobile devices, such as smartphones.

If we can detect the difference between two business process models, we can quickly build a new model by adjusting the existing one. The paper entitled "Detecting Difference between Process Models Based on the Refined Process Structure Tree" by J. Fan et al. presents a new approach that detects the difference of the entire composition. Firstly, it parses the process models to their corresponding 
refined process structure trees (PSTs). Then it converts the PSTs into their corresponding task based process structure trees (TPSTs). As a consequence, the problem of detecting differences between two process models is transformed to detect difference between two TPSTs. Finally, it obtains the differences between two TPSTs based on the divide-andconquer strategy, where the differences are described by an edit script and it makes the cost of the edit script close to minimum.

New information technologies including sensors, indoor positioning, augmented reality, vision, and interactive systems should be utilized by retailers in order to improve operational efficiency and customer experience. The paper entitled "Use of the Smart Store for Persuasive Marketing and Immersive Customer Experiences: A Case Study of Korean Apparel Enterprise" by H. Hwangbo et al. employs the term "smart store" to indicate retail stores equipped with these new technologies and modern marketing concepts. They summarize discussions related to smart stores and their possible applications in a real business environment. Furthermore, they present a case study of a business that applies the smart store concept to its fashion retail shops in Korea.

\section{Acknowledgments}

Yim's work was supported by Small and Medium Business Association (C0443742), by Dongguk University Research Fund, and by the Ministry of Knowledge Economy (10037393).

Jaegeol Yim

Subramaniam Ganesan Byeong Ho Kang 

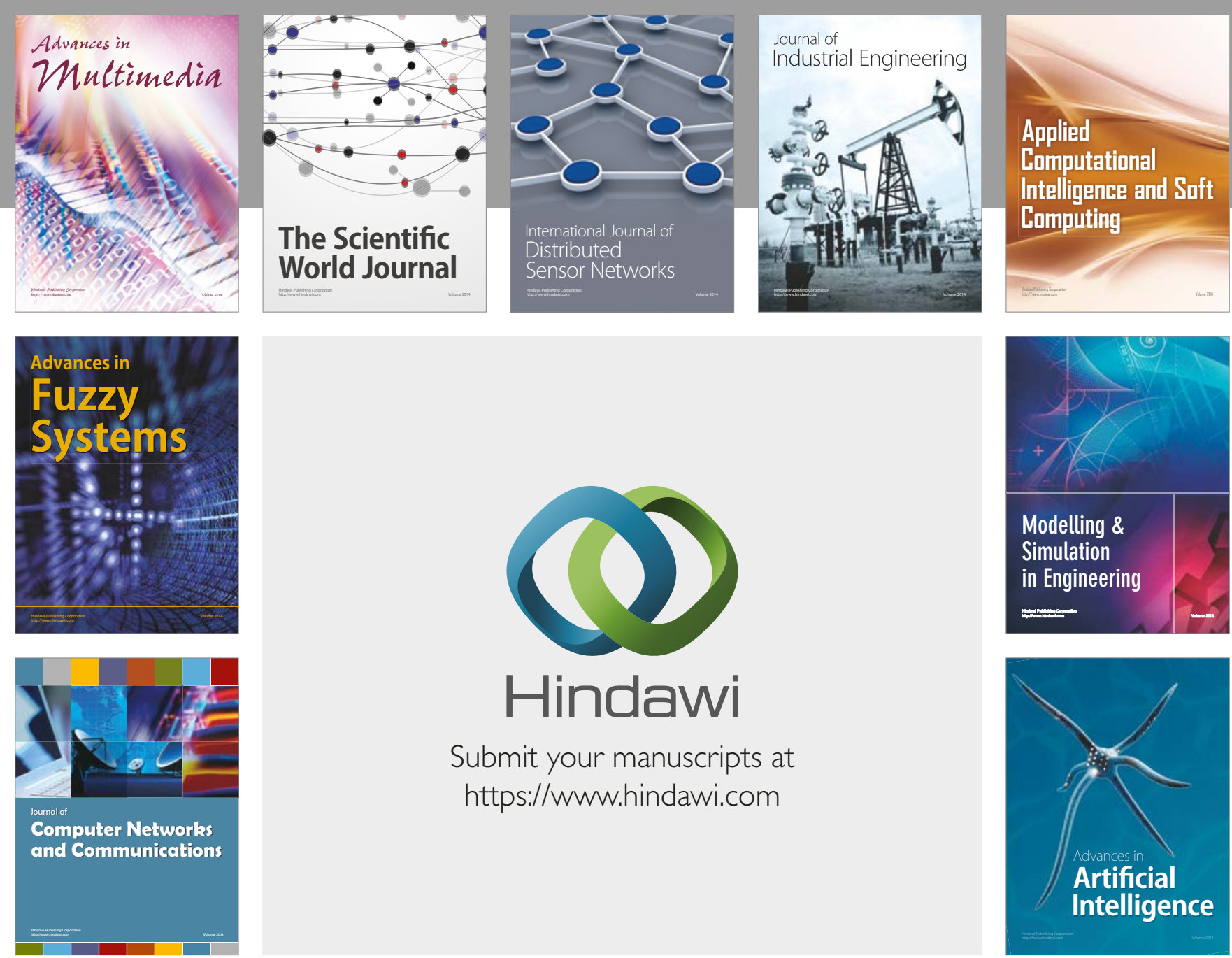

\section{Hindawi}

Submit your manuscripts at

https://www.hindawi.com
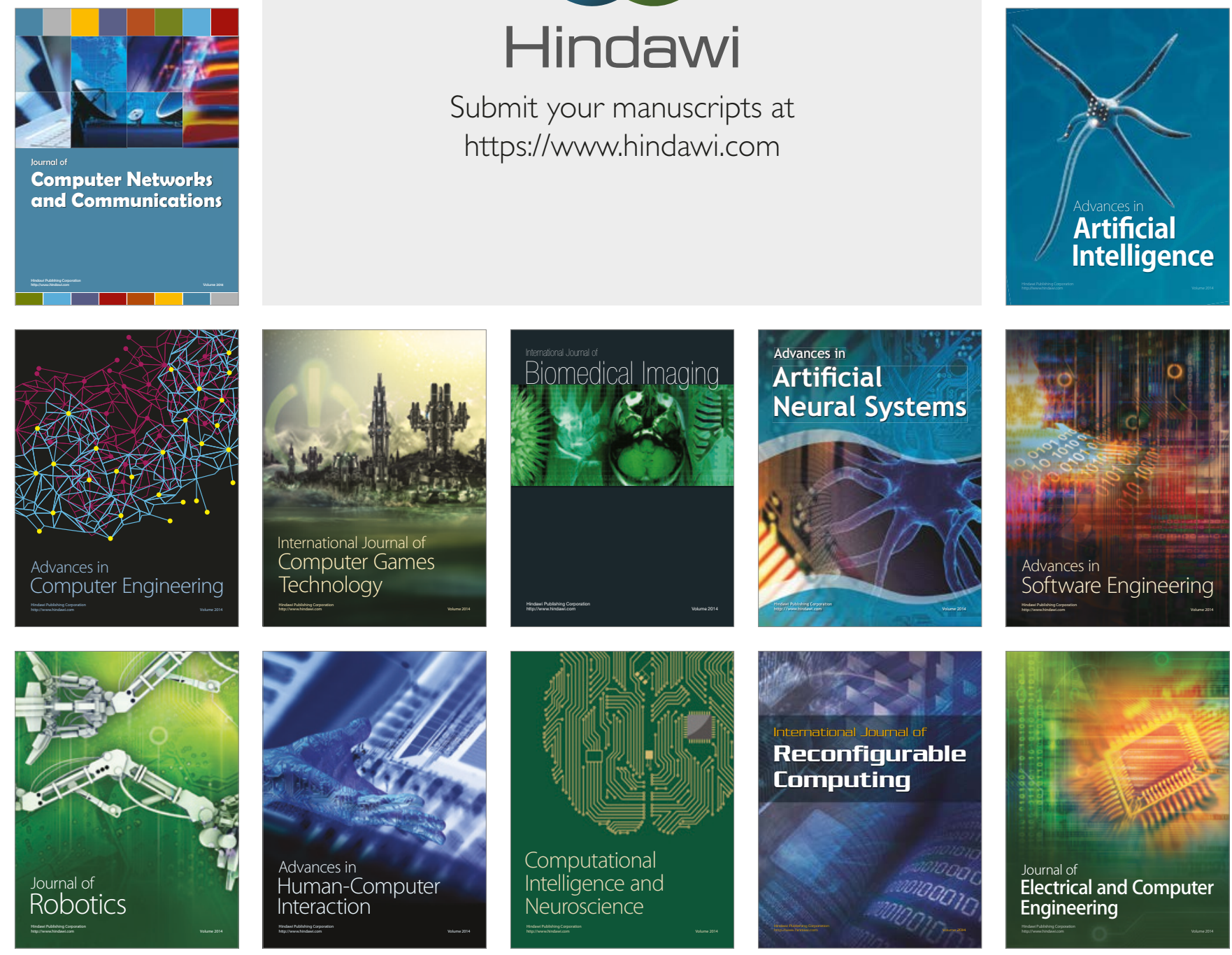